PROCEEDINGS OF THE

AMERICAN MATHEMATICAL SOCIETY

Volume 129, Number 9, Pages 2643-2646

S 0002-9939(01)05858-0

Article electronically published on February 9, 2001

\title{
AN INFINITELY GENERATED INTERSECTION OF GEOMETRICALLY FINITE HYPERBOLIC GROUPS
}

\author{
PERRY SUSSKIND \\ (Communicated by Jozef Dodziuk) \\ In memory of my parents
}

\begin{abstract}
Two discrete, geometrically finite subgroups of the isometries of hyperbolic n-space $(n \geq 4)$ are defined whose intersection is infinitely generated. This settles, in dimensions 4 and above, a long-standing question in Kleinian and hyperbolic groups reiterated at a problem session chaired by Bernard Maskit at the AMS meeting 898, March 3-5, 1995, a conference in honor of Bernard Maskit's 60th birthday.
\end{abstract}

\section{INTRODUCTION}

In 1960, as a generalization of a theorem of Howson [Ho], Greenberg [Gr] proved that if $J$ and $H$ are finitely generated subgroups of a Fuchsian group, then $J \cap H$ is finitely generated. For Kleinian groups (dimensions 2, 3) and hyperbolic groups (discrete subgroups of the isometry group of hyperbolic $n$-space, $n \geq 2$ ), generalizations of the above came along in the 1980's and 90's ([Su,, $\mathrm{SuSw}])$ where the stronger condition (equivalent in dimension 2) of geometric finiteness replaces that of finitely generated. To wit: If $J$ and $H$ are geometrically finite subgroups of a hyperbolic group, then $J \cap H$ is geometrically finite. These results are part of a large stable of theorems in which two subgroups $J$ and $H$ of a hyperbolic group, each possessing some finiteness property (e.g., analytically finite component subgroups, finitely generated Fuchsian or Kleinian groups, geometrically finite hyperbolic groups, function subgroups, topologically tame Kleinian subgroups - see [An2] for a useful survey and bibliography), have an intersection, $J \cap H$, possessing some finiteness property or in which the limit set of $J \cap H$ is, with the possible exception of some isolated points, equal to the intersection of the limit sets of $J$ and $H$.

In the theorems stated above, the proofs require, in an essential way, the hypothesis that the groups $J$ and $H$ together lie inside a (possibly larger) discrete group. The question of whether this hypothesis is necessary in these (and other similar) theorems has been asked (see $[\mathrm{Ba} \mid \underline{\mathrm{Su}}]$ ), yet there appears to be no example in the literature that shows that it is. Indeed, several authors cited in the references have attempted to resolve this question. While the problem remains open in dimensions 2 (Fuchsian case) and 3 (Kleinian case) the following example, which

Received by the editors January 7, 2000.

1991 Mathematics Subject Classification. Primary 30F40; Secondary $20 H 10$.

Key words and phrases. Hyperbolic groups, geometrically finite.

The author thanks William Abikoff and Andrew Haas for reading drafts of this note.

(C)2001 American Mathematical Society 
makes use of parabolic screw motions not available in lower dimensions, provides two geometrically finite hyperbolic groups in dimension 4 (and higher) whose intersection is infinitely generated (and hence, is not geometrically finite). The use of parabolic screw motions, though not strictly necessary - a loxodromic element with an irrational twist would also suffice (see below) - is motivated by Apanasov who showed $\mathrm{Ap}$ that parabolic fixed points in hyperbolic groups need not have invariant horoballs.

\section{BACKGROUND}

The group of isometries of hyperbolic n-space, $M \ddot{o} b_{n}=I \operatorname{som}\left(\mathbb{H}^{n}\right)$, acts on the upper half-space $\mathbb{H}^{n}=\left\{\left(x_{1}, x_{2}, \ldots, x_{n-1}, t\right) \in \mathbb{R}^{n} \mid t>0\right\}$ endowed with the standard metric of constant negative curvature, -1 , and also acts on the boundary $\partial \mathbb{H}^{n}=\mathbb{R}^{n-1} \cup\{\infty\}$ as a group of conformal homeomorphisms. An element $g \neq i d$ of $\operatorname{Isom}\left(\mathbb{H}^{n}\right)$ is elliptic if it has a fixed point in $\mathbb{H}^{n}$. If $g$ is not elliptic and has exactly one fixed point in $\partial \mathbb{H}^{n}$, then $g$ is parabolic; otherwise, $g$ is loxodromic. A subgroup $G$ of $I \operatorname{som}\left(\mathbb{H}^{n}\right)$ is a hyperbolic group if $G$ is discrete. $G$ is discrete if and only if $G$ acts discontinuously on $\mathbb{H}^{n}$. If $g$ is an elliptic element in a hyperbolic group, then $g$ has finite order. The regular set, $\Omega(G)$, is the subset of $\partial H^{n}$ on which $G$ acts discontinuously. The limit set of $G, \Lambda(G)$, is the complement in $\partial \mathbb{H}^{n}$ of $\Omega(G)$. A commonly used characterization of a geometrically finite group, $G$, which holds in all dimensions, is: the thick part of the Nielsen convex core of $G$ is compact. This definition, due to Thurston, is one of five equivalent definitions that hold in arbitrary dimension (see [Bo] for a full discussion). In dimensions 2 and 3, this definition is equivalent to the existence of a finite sided fundamental polyhedron for the action of $G$ on $\mathbb{H}^{n}$. In dimension $2, G$ is geometrically finite if and only if $G$ is finitely generated. (See [Ma2] and [Bo] for definitions and details concerning the background above.)

\section{The CONSTRUCTION}

The following construction in the boundary, $\partial \mathbb{H}^{4}$, may be extended to $\mathbb{H}^{4}$ in the standard way. Let $j$, a pure translation, be the parabolic motion fixing $\infty$ given by $(x, y, z) \mapsto(x, y, z+1)$. Let $U^{1}, U^{-1}$ be spheres with centers $\left(0,0, \frac{1}{4}\right)$ and $\left(0,0, \frac{3}{4}\right)$ respectively, each with fixed radius $r, 0<r<\frac{1}{4}$. Let $g$ be the isometry of $\mathbb{H}^{4}$ whose action on $\partial \mathbb{H}^{4}$ is given by reflection in $U^{1}$ followed by reflection in $\left\{(x, y, z) \mid z=\frac{1}{2}\right\}$, the perpendicular bisector of the line segment joining the centers $\left(0,0, \frac{1}{4}\right)$ and $\left(0,0, \frac{3}{4}\right)$. Let $J=\langle j, g\rangle$ be the group generated by $j$ and $g$. Note that $g$ maps the outside of $U^{1}$ onto the inside of $U^{-1}$.

Let $i$ be the isometry of $\mathbb{H}^{4}$ given by

$$
\begin{gathered}
(x, y, z) \mapsto\left(x^{\prime}, y^{\prime}, z\right), \\
\left(\begin{array}{l}
x^{\prime} \\
y^{\prime}
\end{array}\right)=\left(\begin{array}{cc}
\cos \theta & -\sin \theta \\
\sin \theta & \cos \theta
\end{array}\right)\left(\begin{array}{l}
x \\
y
\end{array}\right),
\end{gathered}
$$

where $\theta$ is an irrational multiple of $\pi$. $i$ acts on $\partial \mathbb{H}^{4}$ as an irrational rotation about the $z$-axis. Let $h=j i$. Put $H=\langle h, g\rangle$. Note that $\Lambda(J)=\Lambda(H) \subset Z=$ $\{(0,0, z) \mid z \in \mathbb{R}\} \cup\{\infty\}, J$ and $H$ leave $Z$ invariant, $j$ and $h$ have the same action on $Z$ and $i$ commutes with $j, g$, and $h$. 
An easy argument (e.g., use the Klein combination theorem [Ma2]) shows that both $J$ and $H$ are discrete and free. Since $J$ and $H$ leave $Z$ invariant, $J$ and $H$ act on the two dimensional hyperplane $\{(0,0, z, t) \mid z \in \mathbb{R}, t>0\}$. Thus, $J$ and $H$ may be regarded as (identical) Fuchsian groups acting on this copy of $\mathbb{H}^{2}$. Since in dimension 2 geometric finiteness is equivalent to discrete and finitely generated, $J$ and $H$ are geometrically finite.

Theorem 1. Let $J$ and $H$ be as above. Then:

(1) $G=\langle j, h, g\rangle$ is not discrete,

(2) $j^{n} g j^{-n}=h^{n} g h^{-n}$ for all $n \in \mathbb{Z}$,

(3) $J \cap H=\left\langle j^{n} g j^{-n} \mid n \in \mathbb{Z}\right\rangle$ is infinitely generated, free, and is free on the generators $j^{n} g j^{-n}=h^{n} g h^{-n}, n \in \mathbb{Z}$.

Proof. For (1) it suffices to note that $G$ contains the elliptic element $i=h j^{-1}$ of infinite order. Since $i$ commutes with $g$ and $j$ it is immediate that $j^{n} g j^{-n}=h^{n} g h^{-n}$ for all $n \in \mathbb{Z}$, proving (2).

For (3), suppose that

$$
\gamma=j^{n_{1}} g^{m_{1}} j^{n_{2}} g^{m_{2}} \ldots j^{n_{s}} g^{m_{s}}=h^{p_{1}} g^{q_{1}} h^{p_{2}} g^{q_{2}} \ldots h^{p_{t}} g^{q_{t}} \in J \cap H .
$$

Using the commutativity of $i$ again,

$$
h^{p_{1}} g^{q_{1}} h^{p_{2}} g^{q_{2}} \ldots h^{p_{t}} g^{q_{t}}=j^{p_{1}} g^{q_{1}} j^{p_{2}} g^{q_{2}} \ldots j^{p_{t}} g^{q_{t}} i^{\left(\sum_{i=1}^{t} p_{i}\right)} .
$$

Since $j$ and $g$ preserve each half-plane in $\partial \mathbb{H}^{4}$ which bounds $Z$, it follows immediately that $\sum_{i=1}^{t} p_{i}=0$. Therefore, $\gamma$ may be written as

$$
\gamma=\left(j^{p_{1}} g j^{-p_{1}}\right)^{q_{1}}\left(j^{p_{1}+p_{2}} g j^{-\left(p_{1}+p_{2}\right)}\right)^{q_{2}} \ldots\left(j^{\sum p_{i}} g j^{-\sum p_{1}}\right)^{q_{t}}
$$

and so $J \cap H$ is generated by the elements $j^{n} g j^{-n}, n \in \mathbb{Z}$. Since $J$ and $H$ are free, $J \cap H$ is free. It remains to show that $J \cap H$ is free on the generators $a_{n}=j^{n} g j^{-n}$, $n \in \mathbb{Z}$.

For $n \in \mathbb{Z}$ put $U_{n}^{1}=j^{n}\left(U^{1}\right), U_{n}^{-1}=j^{n}\left(U^{-1}\right) . \quad U_{n}^{1}\left(U_{n}^{-1}\right)$ is the sphere $U^{1}$ $\left(U^{-1}\right)$ translated by $n$ along the z-axis. The spheres $U_{n}^{1}, U_{n}^{-1}, n \in \mathbb{Z}$, are mutually disjoint and any in the collection lies outside any other in the collection. The element $a_{n}^{e}$, where $e \in\{+1,-1\}$, consists of reflection in $U_{n}^{e}$ followed by reflection in the perpendicular bisector of the segment joining the centers of $U_{n}^{1}$ and $U_{n}^{-1}$. As above one easily sees that the outside of $U_{n}^{e}$ is mapped to the inside of $U_{n}^{-e}$ by the element $a_{n}^{e}$. The following standard argument shows that $J \cap H$ is free on the generators $a_{n}, n \in \mathbb{Z}$. Let $p$ be a point lying outside of all of the spheres $U_{n}^{1}, U_{n}^{-1}$, $n \in \mathbb{Z}$. Suppose $\gamma=a_{n_{1}}^{e_{1}} a_{n_{2}}^{e_{2}} \ldots a_{n_{s}}^{e_{s}}, n_{i} \in \mathbb{Z}, e_{i} \in\{+1,-1\}, i=1, \ldots, s$, where if $n_{i}=n_{i+1}, i=1, \ldots, s-1$, then $e_{i}=e_{i+1}$. The point $p$ lies outside $U_{n_{s}}^{e_{s}}$ so that $a_{n_{s}}^{e_{s}}(p)$ lies inside $U_{n_{s}}^{-e_{s}}$. Thus $a_{n_{s}}^{e_{s}}(p)$ lies outside $U_{n_{s-1}}^{e_{s-1}}$ so that $a_{n_{s-1}}^{e_{s-1}} a_{n_{s}}^{e_{s}}(p)$ lies inside $U_{n_{s-1}}^{e_{s-1}}$. Continuing in this way one sees that $\gamma(p)$ lies inside $U_{n_{1}}^{-e_{1}}$. Therefore $\gamma(p) \neq p$ so that $\gamma \neq i d$. Therefore, $J \cap H$ is free on the generators $a_{n}=j^{n} g j^{-n}$, $n \in \mathbb{Z}$.

The construction and proof above do not depend in an essential way on the use of parabolic elements; rather they depend on the existence of an orthogonal direction for the axis of an irrational twist. For example, the construction and proof above remain essentially the same if the element $j$ is taken to be the loxodromic element $(x, y, z) \mapsto(5 x, 5 y, 5 z), U^{1}$ and $U^{-1}$ are the spheres of radius 1 and centers 3 and 
-3 respectively, where $g$, as before, maps the outside of $U^{1}$ onto the inside of $U^{-1}$, and $h=j i$.

The quotients in $\mathbb{H}^{4}$ of $J$ and $H$ are neither cocompact nor finite volume; moreover, $J$ and $H$ are "elementary" hyperbolic groups in the sense that they both leave invariant the same 2-dimensional hyperplane. Are there examples of cocompact, finite volume or nonelementary groups which produce a similar result concerning the need for $\langle J, H\rangle$ to be discrete?

\section{REFERENCES}

[An1] J. W. Anderson, Intersections of analytically and geometrically finite subgroups of Kleinian groups, Trans. Amer. Math. Soc. 343 (1994), 87-98. MR 95d:30083

[An2] J. W. Anderson, The limit set intersection theorem for finitely generated Kleinian groups, Mathematical Research Letters 3 (1996), 675-692. MR 98k:30056

[Ap] B.N. Apanasov, Cusp ends of hyperbolic manifolds, Ann. Global Analysis and Geometry, No. 1, 3 (1985), 1-11. MR 88b:22012

[Ba] A. Basmajian, Selected problems in Kleinian groups and hyperbolic geometry, in Lipa's Legacy, Contemporary Mathematics, vol. 211, Amer. Math. Soc., Providence, RI, 1997, 9-15. MR 98g:30072

[BeMa] A. F. Beardon and B. Maskit, Limit points of Kleinian groups and finite sided polyhedra, Acta. Math. 132 (1974),1-12. MR 48:11489

[Bo] B. H. Bowditch, Geometric finiteness for hyperbolic groups, J. Functional Anal. 113 (2) (1993), 245-317. MR 94e:57016

[Gr] L. Greenberg, Discrete groups of motions, Canadian J. Math. 12 (1960), 415-426. MR 22:5932

[Ho] A. G. Howson, On the intersection of finitely generated free groups, J. Lond. Math. Soc. 29 (1954), 428-434. MR 16:444c

[Ma1] B. Maskit, Intersections of component subgroups of a Kleinian group in Discontinuous Groups and Riemann Surfaces, Annals of Mathematics Studies no. 79, Princeton University Press, 1974. MR 50:7514

[Ma2] B. Maskit, Kleinian Groups, Springer-Verlag, New York, 1988. Amer. Math. Soc., Providence, RI, 1993. MR 90a:30132

[Su] P. Susskind, Kleinian groups with intersecting limit sets, J. d'Analyse Mathematique 52 (1989), 26-38. MR 90f:57045

[SuSw] P. Susskind and G. Swarup, Limit sets of geometrically finite hyperbolic groups, Amer. J. Math. 114 (1992), 233-250. MR 94d:57066

Department of Mathematics, Connecticut College, Box 5596, New London, ConNECTICUT 06320

E-mail address: pdsus@conncoll.edu 